\title{
The impact of frame numbers on cardiac ECG-gated SPECT images with interpolated extra frames using echocardiography
}

\author{
Maghsoud Sarebani ${ }^{1}$, Mohammad Bagher Shiran*1(D), Ahmad Bitarafan-Rajabi*2,3(D), Feridoon Rastgou ${ }^{4}$, \\ Zahra Ojaghi Haghighi ${ }^{5}$, Ali Abbasian Ardakani ${ }^{1}$
}

Received: 7 Oct 2018

Published: 3 Jun 2020

\section{Abstract}

Background: Cardiac echocardiography and cardiac ECG-gated single-photon emission computed tomography (SPECT) are the most common modalities for left ventricle (LV) volumes and function assessment. The temporal resolution of SPECT images is limited and an ECG provides better temporal resolution. This study investigates the impact of frame numbers on images in terms of qualitative and quantitative assessments.

Methods: In this study, 5 patients underwent echocardiography and cardiac ECG-gated SPECT imaging, and 5 standard views of the LV were recorded to determine LV walls boundaries and volumes. Also, 2 original images with 8 frames and 16 frames per cardiac cycle were recorded simultaneously in a single gantry orbit. Using the data extracted from the LV model, 8 extra new frames were created with interpolation between existing frames of the original 8-frame image. Three series of images ( 8 and 16 original and 16 interpolated) were reconstructed separately. LV volumes and ejection fraction (EF) were calculated using Quantitative Gated SPECT (QGS) software.

Results: Compared to the original 8-frame gating, original 16-frame gated images resulted in larger end-diastole volume (EDV) (mean \pm SD: $68.6 \pm 27.11 \mathrm{~mL}$ vs $66.2 \pm 25.41 \mathrm{~mL}, \mathrm{p}<0.001$ ), smaller end-systole volume (ESV) (mean \pm SD: $24.6 \pm 8.7 \mathrm{~mL}$ vs $26 \pm 7.3 \mathrm{~mL}$, $\mathrm{p}<0.001)$, and higher EF ( $64 \%$ vs $60.2 \%, \mathrm{p}<0.001)$. The results for the interpolated series were also different from the original images (closer to the original 16-frame series rather than 8 -frame).

Conclusion: Changing the frame number from 8 to 16 in cardiac ECG-gated SPECT images caused a significant change in LV volumes and EF. Frame interpolation with sophisticated algorithms can be used to improve the temporal resolution of SPECT images.

Keywords: SPECT, Echocardiography, Interpolation, End-diastole volume (EDV), End-systole volume (ESV), Ejection fraction (EF)

Conflicts of Interest: None declared

Funding: This study was funded by Iran University of Medical Sciences (Grant \# 96-01-30-29288).

\section{*This work has been published under CC BY-NC-SA 1.0 license. \\ Copyright $\odot$ Iran University of Medical Sciences}

Cite this article as: Sarebani M, Shiran MB, Bitarafan-Rajabi A, Rastgou F, Ojaghi Haghighi Z, Abbasian Ardakani A. The impact of frame numbers on cardiac ECG-gated SPECT images with interpolated extra frames using echocardiography. Med J Islam Repub Iran. 2020 (3 Jun);34:57. https://doi.org/10.47176/mjiri.34.57

\section{Introduction}

Heart diseases are the leading cause of death in most developing countries (1). Early and accurate diagnosis is the

1. Department of Medical Physics, School of Medicine, Iran University of Medical Sciences, Tehran, Iran

2. Cardiovascular Intervention Research Center, Rajaie Cardiovascular Medical and Research Center, Iran University of Medical Sciences, Tehran, Iran

3. Echocardiography Research Center, Rajaie Cardiovascular Medical and Research Center, Iran University of Medical Sciences, Tehran, Iran

4. Rajaie Cardiovascular Medical and Research Center, Iran University of Medical Sciences, Tehran, Iran

5. Department of Echocardiography, Rajaei Cardiovascular, Medical and Research Center, Iran University of Medical Science, Tehran, Iran

key in the successful treatment of heart problems (2). In nu-

$\uparrow$ What is "already known" in this topic:

ECG-gated single-photon emission computed tomography (SPECT) is used to improve temporal resolution and functional assessment of the left ventricle (LV).

\section{$\rightarrow$ What this article adds:}

This study aimed to use frame interpolation techniques to create accurate frames in cardiac-gated SPECT images and to help improve temporal resolution. The results revealed that 16-frame images provide better temporal resolution extracted data from echocardiography and could be used to improve temporal resolution in SPECT images and reduce imaging time. In this regard, proposed methods can be used to evaluate cardiac function more accurately. 
clear medicine, ECG-gated single-photon emission computed tomography (SPECT) provides a functional assessment of the left ventricle (LV) (3). To improve temporal resolution, data acquisition during a cardiac cycle is divided into several frames using this technique. Each frame represents the LV in the related time interval during the cardiac cycle. In clinical applications, 16-frame or 8-frame images are used. A higher number of frames results in poorer quality of each frame. This is because of the low signal to noise ratio (SNR). The impact of frame numbers is a controversial issue both from the point of view of clinical benefits and image quality (4). Echocardiography, due to the physics of ultrasound, provides better temporal resolution than nuclear medicine (about 30-60 frames). Therefore, in ultrasound images, we can get information at shorter time intervals, which are missing in SPECT images (5). Image processing as the art of image manipulation is very useful in obtaining more information from an existing image or representing it in a more useful way. Image interpolation is one of the most important techniques in image processing. One of the applications of image interpolation is to create missing frames of a recorded dynamic event using the existing frames (6). This technique is widely used in video processing and data storage processes. Using frame interpolation techniques to create accurate frames in cardiac-gated SPECT images can help to improve temporal resolution. This study aimed to investigate the impact of frame numbers in cardiac-gated SPECT images from the perspective of both image quality and clinical benefit. In addition, in this study, the possibility of using image interpolation technique was investigated to increase the frame numbers in cardiac-gated SPECT images.

\section{Methods \\ Echocardiography and cardiac nuclear medicine exam- ination}

This study was approved by a local ethics committee and informed consent was obtained from all patients. The 5 patients who were recommended to take myocardial perfusion imaging (MPI) with SPECT underwent common SPECT examination. They were injected with $740 \mathrm{MBq}{ }^{99 \mathrm{~m} T \mathrm{c}-}$ MIBI. MPI was performed using a dual head SPECT machine. This machine provides the possibility of implementation of 2 separate protocols for data recording, storage, and process in a single acquisition. To investigate the effect of frame number on images, 2 separate protocols were used, which were identical in all parameters except in frame per cardiac cycle. Some parameters included $64 * 64$ matrix size, $6.4 \mathrm{~mm}$ pixel dimensions, 1.45 zoom factor, and 16 stops ( 32 projections for 2 heads); $20 \%$ energy window was the same for both protocols. ECG-gated cardiac images were acquired with 8 frames and 16 frames per R-R intervals. The 8 -frame series of images have 8 missing frames compared to the 16-frame series; we call them missing frames. A cardiologist conducted a 2D cardiac ultrasound imaging examination on all patients. Five standard views of the heart were recorded using a Philips HD11 machine. Three short axis (SA: epical - mid - base), apical 2-chamber (A2C), and apical 4-chamber (A4C) views (Fig. 1).

The boundaries of the LV walls were determined and marked by the cardiologist. The volumetric process was performed to estimate the LV volumes and function. The marked wall boundaries and volumetric data were extracted for further processes to model the LV.

\section{Ultrasound and SPECT images time registration}

Cardiac US images were also gated using ECG R-R intervals (48 frames). It is possible to relate each SPECT frame to the proportional frame of US images when both are representing LV in a specific time slot during the cardiac cycle.

\section{Modeling of LV and its location in SPECT projections}

To estimate an approximation of location of pixels, which are representing the LV in SPECT projections, LV geometry and its location in SPECT imaging were modeled. The boundary walls were extracted from US images. Five cross section planes were used to reconstruct a volume as LV (Fig. 2).

The geometry and volumetric data from US were used to optimize the algorithm. The modeling process was repeated for each of the 8 missing frames. The produced LV model was used in a 2D Radon transform process to obtain an approximation of SPECT projections. The zoom factor of gamma camera and its field of view and the distance from LV to gamma camera were considered. Each projection was mapped into a $64^{*} 64$ matrix. The pixels with non zero values address the pixels which represent LV on SPECT projections. Interpolation of missing projections for 8frame image series, 8 new frames were created using image

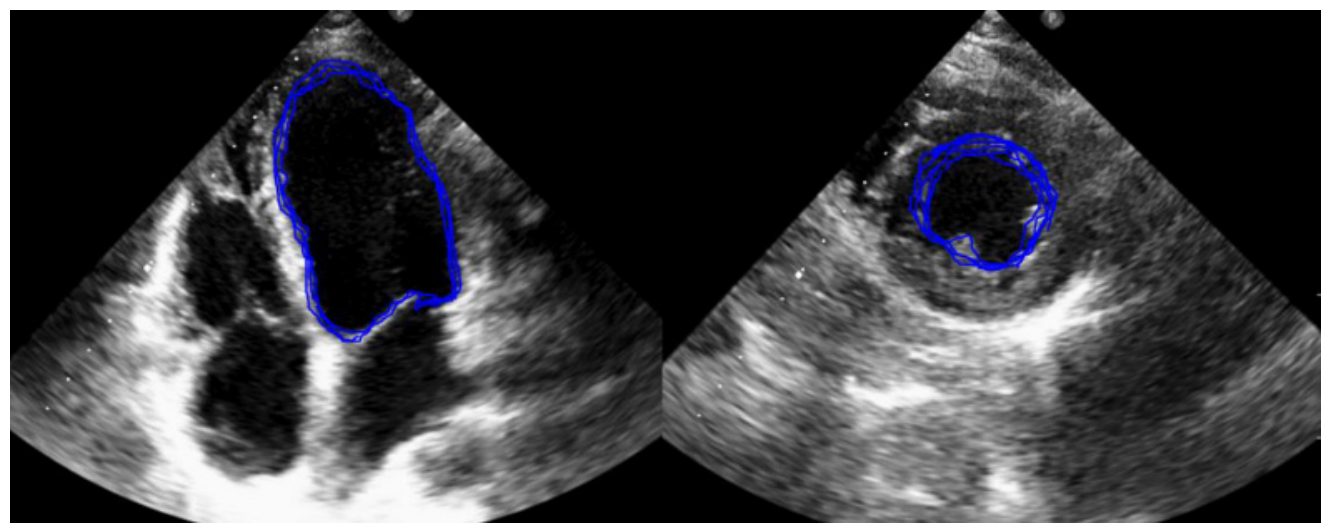

Fig. 1. 2D ultrasound images of left ventricle 


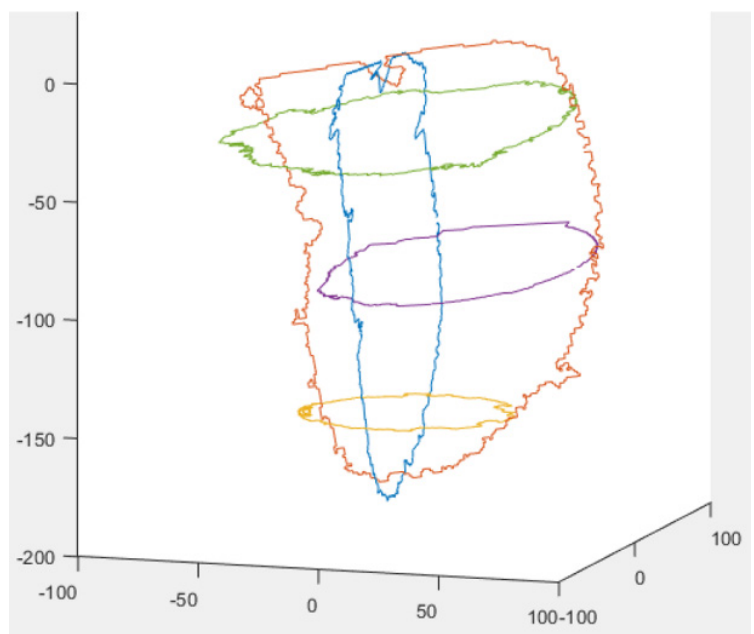

Fig. 2. Left ventricle, reconstructed from cross sectional curves extracted from US images

interpolation techniques. Interpolation was performed to create 1 frame between 2 consecutive frames. Two separate algorithms were applied for 2 separate parts of each image. To maximize the accuracy of interpolation for value of predefined pixels which represent the LV, the cubic spline method was used to interpolate this part of the matrixes. Also, to reduce the number of calculations, linear algorithm was applied to the rest of the pixels so that the impact on the final reconstructed image was lower.

\section{Qualitative and quantitative image comparison}

For each patient, there were 3 series of images, 2 original series (with 16 and 8 frames per cardiac cycle) and an interpolated 16-frame series with 8 original and 8 interpolated frames. Three series of images were reconstructed using standard nuclear medicine protocols and LV quantification process was performed for each series of images (Figs. 3-5). LV volumes and its function were calculated (Figs. 6-8).

A nuclear medicine specialist reviewed the images and their diagnostic values were rated on a scale of 1 to 5 .

\section{Statistical analysis}

All data analysis was performed using SPSS software version 24. A nonparametric Wilcoxon signed ranked test was used to compare the mean values of EDV, ESV, and LVEF. $\mathrm{P}<0.05$ was considered statistically significant.

\section{Results}

The results of this study are summarized in Tables 1 and 2. Using the Wilcoxon signed rank test to compare the mean values, end-diastole volume (EDV) as well as Ejection fraction $(\mathrm{EF})$ were increased $(2.4 \mathrm{~mL}$ and $3.8 \%$, respectively) as the number of frames increased. Increasing the frame numbers from 8 to 16 caused $1.4 \mathrm{~mL}$ reduction in measured end-systole volume (ESV). In qualitative assessments, the images were visually compared. The clinical findings from the original 16-frame series were considered as a standard compared to the interpolated 16-frame and original 8-frame series. The utility of images to address these findings was graded on a 1-5 scale. The average rating for both series was 3.66.

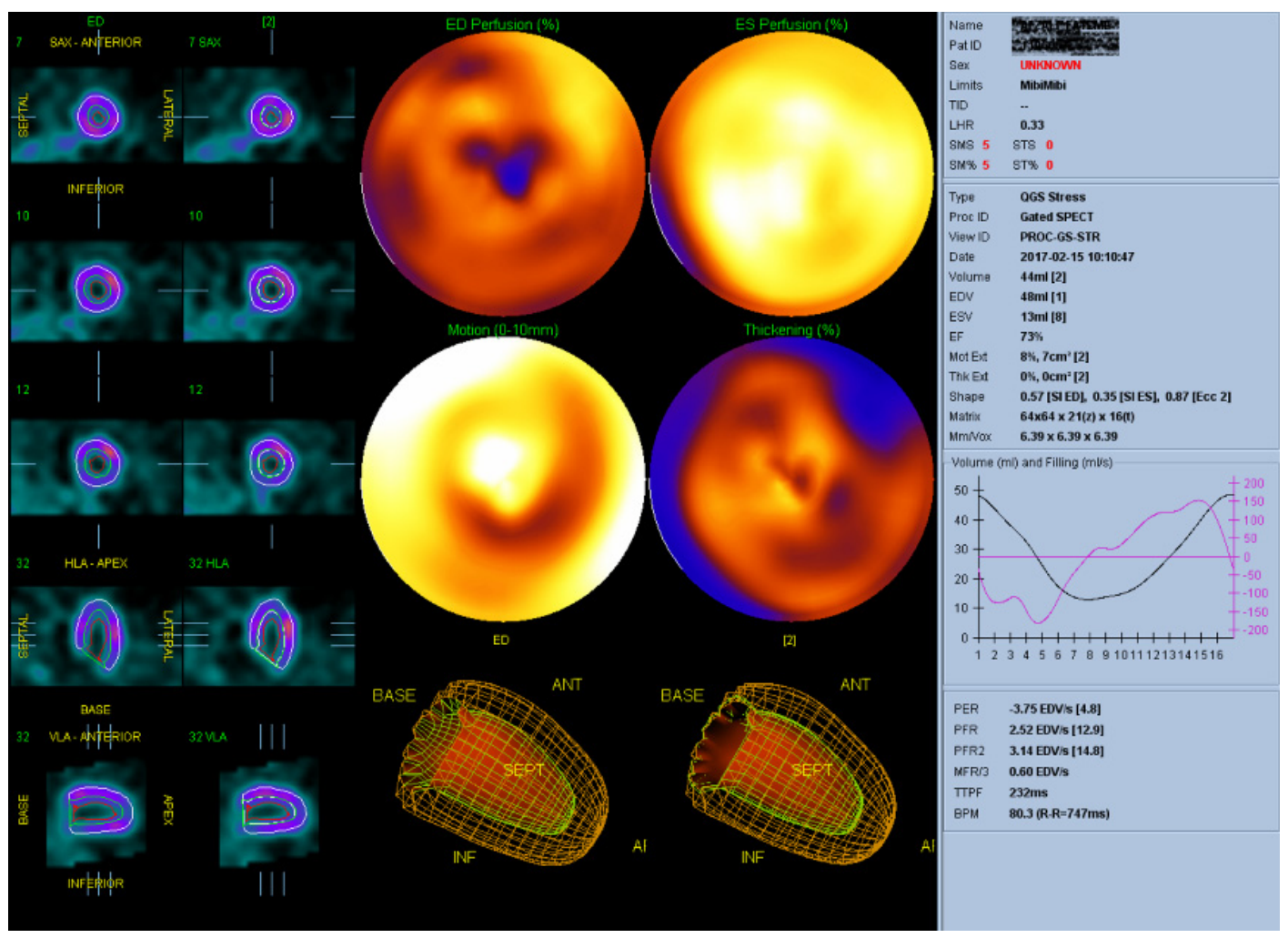

Fig. 3. QGS results for originally 16 -frames image 


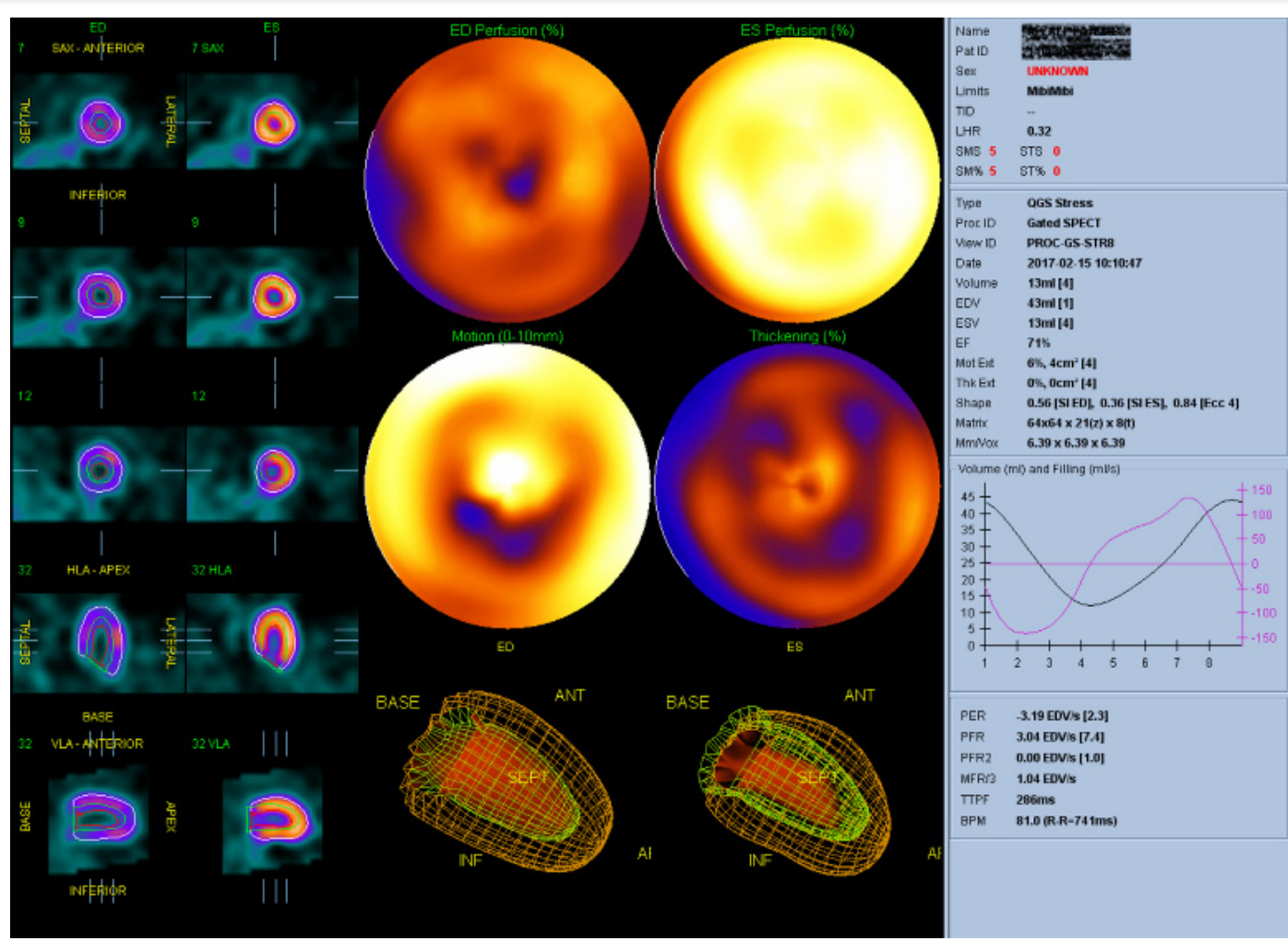

Fig. 4. QGS results for originally 8-frames image

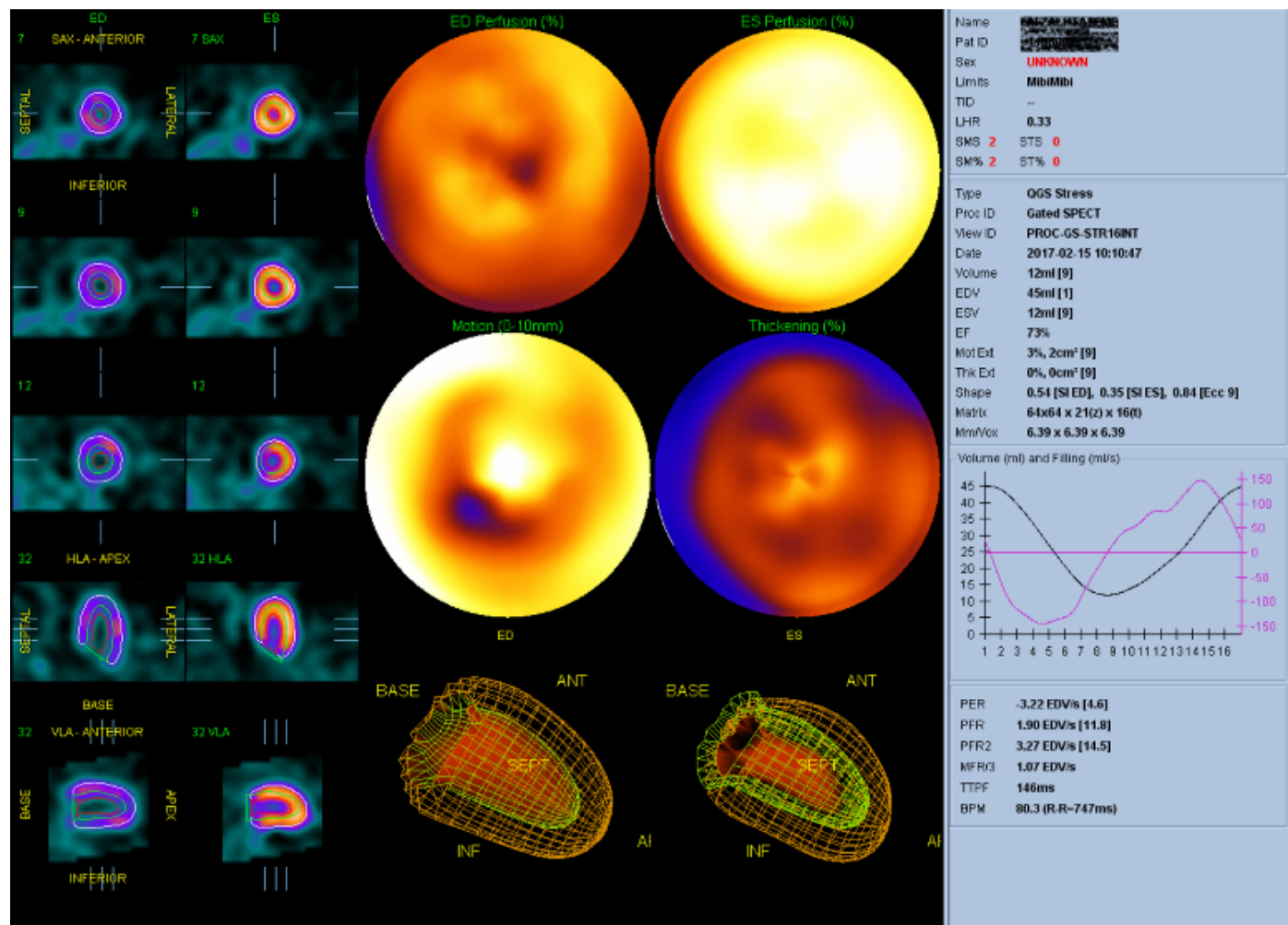

Fig. 5. QGS results for interpolated 16-frames image

\section{Discussion}

Eight-frame and 16-frame images comparison

In this study, 2 important modalities with high diagnostic accuracy (echocardiography and SPECT imaging) and 2 specialist physicians were used as reference in each of the 2 modalities. The results of this study showed that calculated LV volumes from 8-frame images differ from those calculated from 16-frame series of cardiac SPECT images. The mean value of EDV was larger for 16-frame images. In 


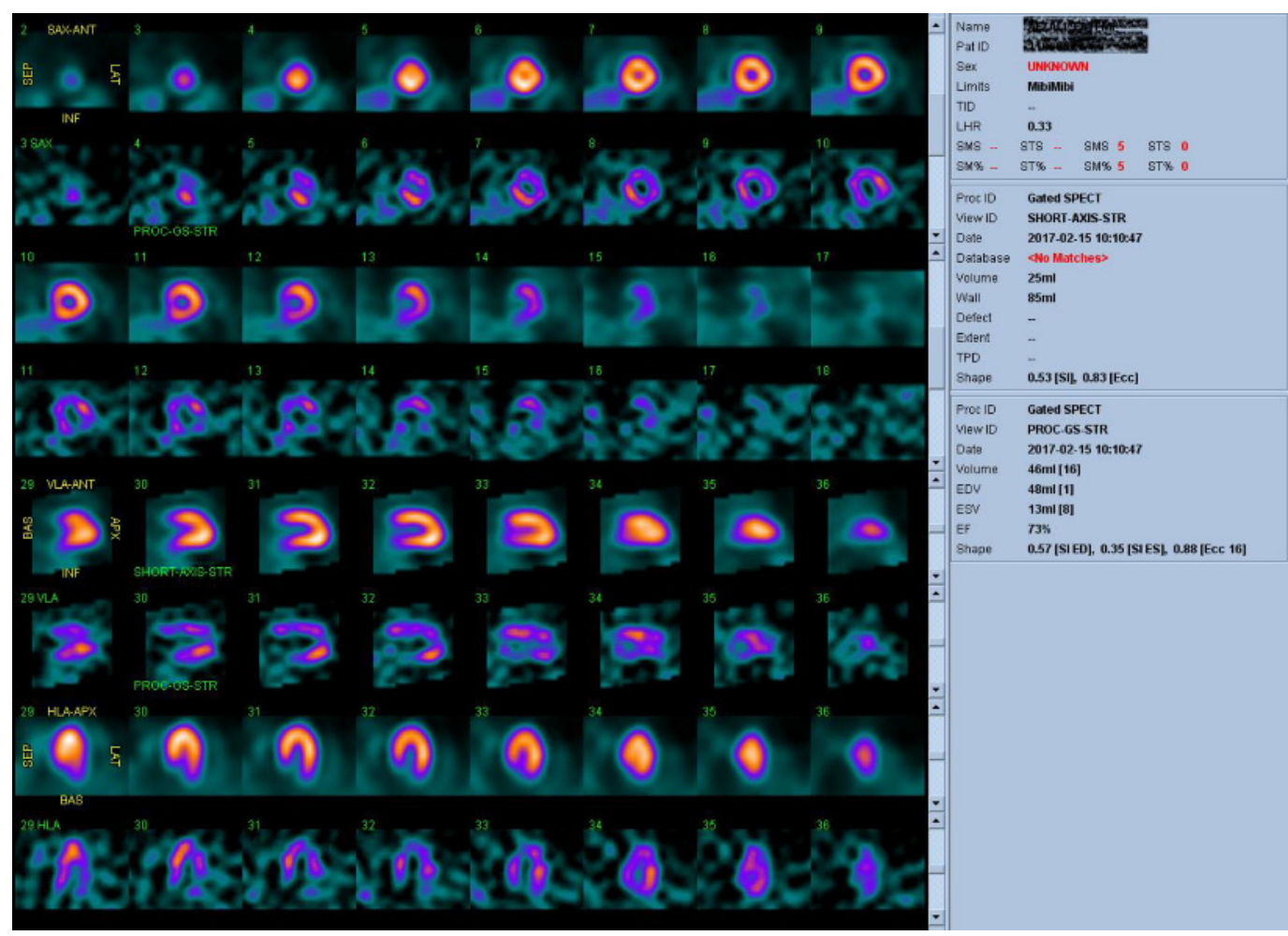

Fig. 6. LV nuclear medicine slices for original 16-frame series

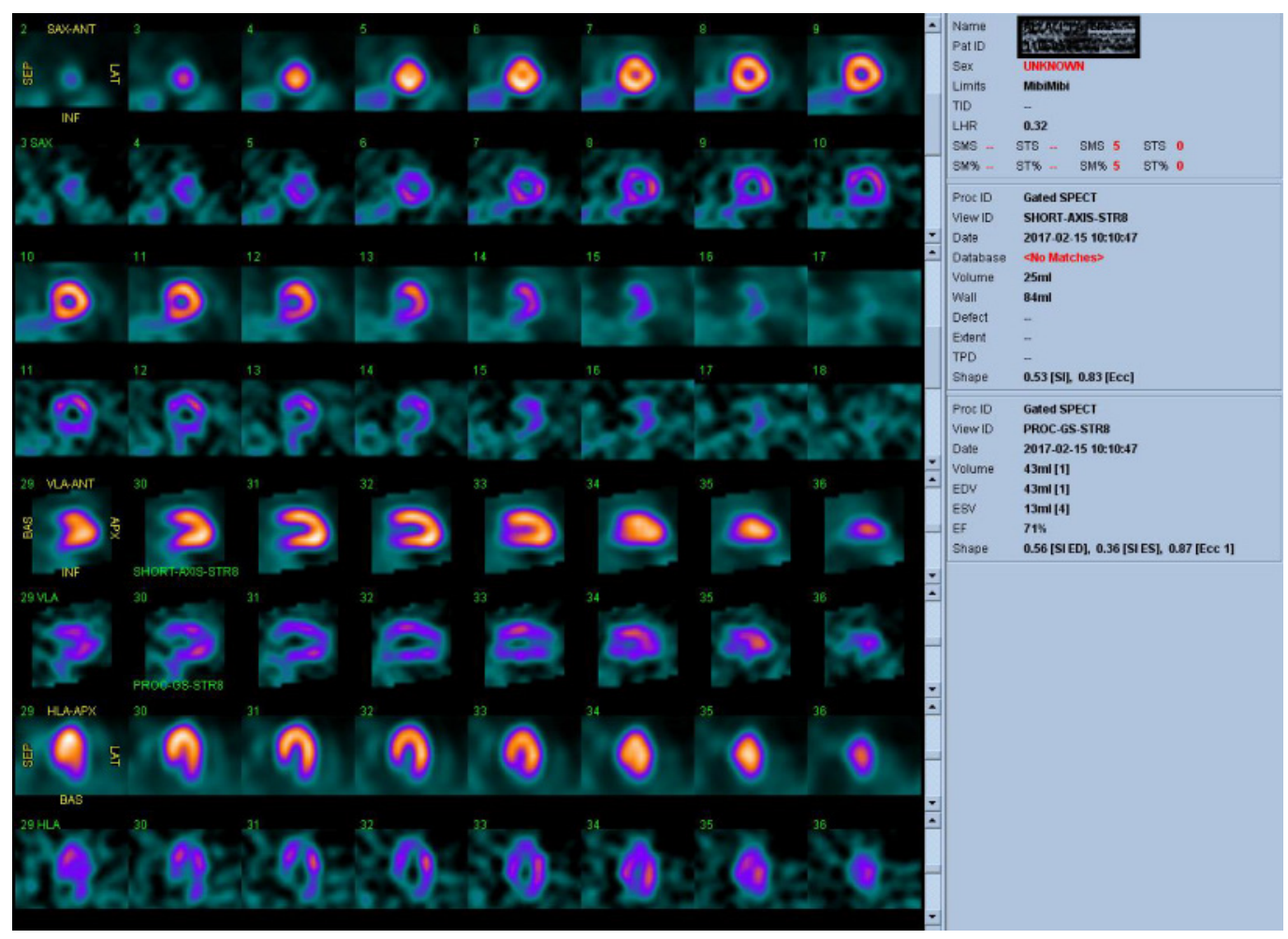

Fig. 7. LV nuclear medicine slices for original 8-frame series

addition, the mean value of ESV for 16-frame series was smaller compared to the 8-frame images. As a consequence of higher EDV and lower ESV, the EF obtained was higher for the 16-frame series. These results agree with the findings of other studies $(4,7,8)$. The difference in calculated volumes can be explained considering the difference in temporal resolution between the 2 series. If the R-R interval is divided into 8 frames in the ECG-Gated SPECT imaging, it will increase the count per frame due to the increase in time for each frame. This means that the information from 


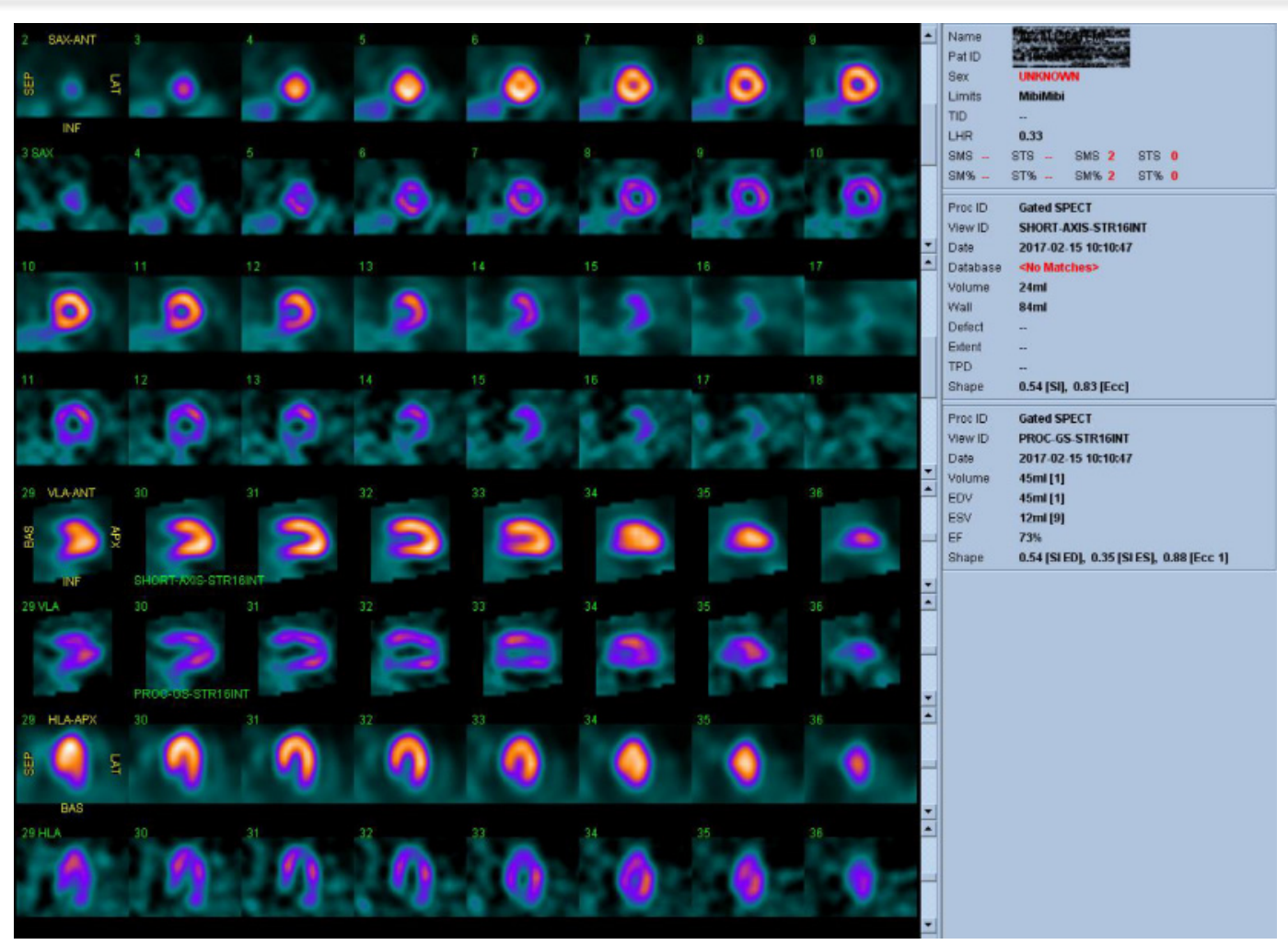

Fig. 8. LV nuclear medicine slices for interpolated 16-frame series

Table 1. Comparison of mean value of echocardiography with 8-frame and 16-frame SPECT

\begin{tabular}{lccccc}
\hline $\begin{array}{l}\text { Cardiac function } \\
\text { parameters }\end{array}$ & \multicolumn{2}{c}{ SPECT } & Echocardiography & $\mathrm{r}$ & P value \\
\hline EDV $(\mathrm{mL})$ & 16-frame & $68.6 \pm 27.11$ & $60.6 \pm 12.8$ & 0.95 & $<0.001$ \\
& 8-frame & $66.2 \pm 25.41$ & $60.6 \pm 12.8$ & 0.91 & $<0.001$ \\
ESV $(\mathrm{mL})$ & 16-frame & $24.6 \pm 8.7$ & $24.8 \pm 9.17$ & 0.94 & $<0.001$ \\
& 8-frame & $26 \pm 7.3$ & $24.8 \pm 9.17$ & 0.93 & $<0.001$ \\
LVEF $(\%)$ & 16-frame & $64 \pm 8.9$ & $60.8 \pm 9.4$ & 0.94 & $<0.001$ \\
& 8-frame & $60.2 \pm 8.4$ & $60.8 \pm 9.4$ & 0.89 & $<0.001$ \\
\hline
\end{tabular}

Values are expressed as mean \pm SD. r, correlation coefficient.

Table 2. Comparison of mean value of SPECT 8-frame and 16-frame images

\begin{tabular}{|c|c|c|c|c|}
\hline $\begin{array}{l}\text { Cardiac function } \\
\text { parameters }\end{array}$ & Original 16-frame & Interpolated 16-frame & $\mathrm{r}$ & $P$ value \\
\hline $\mathrm{EDV}(\mathrm{mL})$ & $68.6 \pm 27.11$ & $67.2 \pm 25.36$ & 0.93 & $<0.001$ \\
\hline $\mathrm{ESV}(\mathrm{mL})$ & $24.6 \pm 8.7$ & $24.2 \pm 5.8$ & 0.92 & $<0.001$ \\
\hline LVEF (\%) & $64 \pm 8.9$ & $63 \pm 9.1$ & 0.93 & $<0.001$ \\
\hline
\end{tabular}

Values are expressed as mean \pm SD. r, correlation coefficient.

the cardiac function parameters such as EF, EDV, and ESV will be more accurately recorded. On the other hand, in the 8 frames, some of the cardiac function information will be miss such as cardiac diastolic function parameters. In this study, however, we tried to convert 8 to 16 frames in addition to compensating for the decrease in counts per imaging frame, increasing the accuracy of estimating the quantitative functional information of the heart. As a result, increased diagnostic accuracy of cardiac function indices will be of great importance in interpreting a nuclear medicine specialist and a cardiologist to continue treatment. In addition, in the conventional 16-frame method, it is necessary to increase the imaging time to obtain the appropriate count, which will increase the total imaging time and the resulting problems such as motion artefacts. Hence, the greater the number of frames, the more temporal resolution and thin time slots for each frame. The 16-frame series, which has superior temporal resolution and shorter time intervals, represents LV closer to the real end-systolic and end-diastolic points. This results in smaller ESV and larger EDV.

Also, the LV volumes and EF derived from SPECT images were not compared with a gold standard modality. However, previous studies reported underestimated EDV and EF in an 8-frame series as compared to other standard modalities (9-11). Thus, the results of the 16-frame series are more comparable with the gold standard. Also, in this study, the presence of perfusion defects was not considered as a parameter to be compared in images. In some studies, an overestimation in LV function due to myocardial perfusion defects has been reported (12). At the same time, there are others that have emphasized that perfusion defects have 
no effect on volume estimations $(8,13,14)$. Manrique et al reported underestimated LVEF in patients with significant perfusion defects in SPECT images (15). Navare et al reported that the results of $\mathrm{LV}$ volumes comparison between the 8-frame and 16-frame series are also independent from injected radiotracer doses (8).

\section{6-frame interpolated images}

In this study, 8 new frames were created between the consecutive frames of the original 8 -frame acquired image. The interpolated 16-frame images represent cardiac function better than the original 8-frame images but are not as good as the original 16-frame images. The visual evaluation of reconstructed images revealed that in some cases the 8frame images exaggerate diastolic dysfunction which has been regulated in interpolated 16-frame images. In quantitative assessment, the measured volumes from interpolated images are closer to those measured from the original 16frame rather than the original 8-frame images.

In this study, some approximations were considered in the modeling process (explained in material and method). Also, there were some limitations which affected the results. This study was designed to investigate the possibility of using interpolation to create more frames for SPECT images. However, the results are not reliable from a statistical point of view as only a few samples were studied. One of the limitations was that the post stress SPECT images were repaired using rest echocardiography images. Another limitation was that in this study the original 8-frame and 16frame images were obtained simultaneously (with the same total count) during a single gantry orbiting. Separate acquisitions with lower dose and time for 8-frame images can lead to a more accurate comparison.

This study showed that measured volumes and EF from 16-frame cardiac-gated SPECT images are significantly different from those provided by 8 -frame images. Also, a 16-frame image provides better temporal resolution compared to an 8-frame image. Thus, the 16-frame per cycle may be the preferred gating protocol for cardiac perfusion imaging. Also, with more accurate algorithms, the interpolation technique can be used to create further frames to improve temporal resolution.

\section{Conclusion}

We performed ECG-gated myocardial perfusion SPECT to acquire images with 8-frame and 16-frame original frame per cardiac cycle and quantified them to obtain LV volumes and $\mathrm{EF}$. The results were compared to investigate the impact of the number of frames on images and LV function. The interpolation technique was implanted to create 8 more frames in the original 8 frame images. The results from interpolated images were then compared to the original images. The results revealed that 16 -frame images provide better temporal resolution extracted data from echocardiography and could be used to improve temporal resolution in SPECT images.

\section{Acknowledgements}

This study was funded by Iran University of Medical Sciences (Grant \# 96-01-30-29288).

\section{Conflict of Interests}

The authors declare that they have no competing interests.

\section{References}

1. Gaziano TA, Bitton A, Anand S, Abrahams-Gessel S, Murphy A. Growing epidemic of coronary heart disease in low-and middle-income countries. Curr Probl Cardiol. 2010;35(2):72-115.

2. Carney RM, Rich MW, Freedland KE, Saini J, teVelde A, Simeone C, et al. Major depressive disorder predicts cardiac events in patients with coronary artery disease. Psychosomatic Med. 1988.

3. Singh B, Bateman TM, Case JA, Heller G. Attenuation artifact, attenuation correction, and the future of myocardial perfusion SPECT. J Nucl Cardiol. 2007;14(2):153-64.

4. Montelatici G, Sciagrà R, Passeri A, Dona M, Pupi A. Is 16-frame really superior to 8-frame gated SPECT for the assessment of left ventricular volumes and ejection fraction? Comparison of two simultaneously acquired gated SPECT studies. Eur J Nucl Med Mol Imaging. 2008;35(11):2059-65.

5. Himelman RB, Cassidy MM, Landzberg JS, Schiller NB. Reproducibility of quantitative two-dimensional echocardiography. Am Heart J. 1988;115(2):425-31.

6. Lehmann TM, Gonner C, Spitzer K. Survey: Interpolation methods in medical image processing. IEEE Trans Med Imaging. 1999;18(11):1049-75.

7. Kumita SI, Cho K, Nakajo H, Toba M, Uwamori M, Mizumura S, et al. Assessment of left ventricular diastolic function with electrocardiography-gated myocardial perfusion SPECT: comparison with multigated equilibrium radionuclide angiography. J Nucl Cardiol. 2001;8(5):568-74.

8. Navare SM, Wackers FJ, Liu YH. Comparison of 16-frame and 8-frame gated SPET imaging for determination of left ventricular volumes and ejection fraction. Eur J Nucl Med Mol Imaging. 2003;30(10):1330-7.

9. Vaduganathan P, He ZX, Vick GW, Mahmarian JJ, Verani MS Evaluation of left ventricular wall motion, volumes, and ejection fraction by gated myocardial tomography with technetium 99 m-labeled tetrofosmin: a comparison with cine magnetic resonance imaging. J Nucl Cardiol. 1999;6(1):3-10.

10. Yoshioka J, Hasegawa S, Yamaguchi H, Tokita N. Left ventricular volumes and ejection fraction calculated from quantitative electrocardiographic-gated $99 \mathrm{mTc}$-tetrofosmin myocardial SPECT. J Nucl Med. 1999;40(10):1693.

11. Nichols K, Tamis J, DePuey EG, Mieres J, Malhotra S, Rozanski A. Relationship of gated SPECT ventricular function parameters to angiographic measurements. J Nucl Cardiol. 1998;5(3):295-303.

12. Vallejo E, Dione DP, Bruni WL, Constable RT. Reproducibility and accuracy of gated SPECT for determination of left ventricular volumes and ejection fraction: experimental validation using MRI. J Nucl Med. 2000;41(5):874.

13. Everaert H, Bossuyt A, Franken PR. Left ventricular ejection fraction and volumes from gated single photon emission tomographic myocardial perfusion images: comparison between two algorithms working in three-dimensional space. J Nucl Cardiol. 1997;4(6):472-6.

14. Chua T, Yin LC, Thiang TH, Choo TB, Ping DZ, Leng LY. Accuracy of the automated assessment of left ventricular function with gated perfusion SPECT in the presence of perfusion defects and left ventricular dysfunction: correlation with equilibrium radionuclide ventriculography and echocardiography. J Nucl Cardiol. 2000;7(4):301-11.

15. Manrique A, Faraggi M, Vera P, Vilain D. $201 \mathrm{~T} 1$ and $99 \mathrm{mTc}-\mathrm{MIBI}$ gated SPECT in patients with large perfusion defects and left ventricular dysfunction: comparison with equilibrium radionuclide angiography. J Nucl Med. 1999;40(5):805. 